\title{
Nondestructive determination of IMP content in chilled chicken based on hyperspectral data combined with chemometrics
}

\author{
Yangyang Wang, Hongju He*, Shengqi Jiang, Hanjun Ma \\ (School of Food Science, Henan Institute of Science and Technology, Xinxiang 453003, Henan, China)
}

\begin{abstract}
This study was conducted to investigate the potential of hyperspectral imaging technique (900-1700 nm) for nondestructive determination of inosinic acid (IMP) in chicken. Hyperspectral images of chicken flesh samples were acquired, and their mean spectra within the images were extracted. The quantitative relationship between the mean spectra and reference IMP value was fitted by partial least squares (PLS) regression algorithm. A PLS model (MAS-PLS) built with moving average smoothing (MAS) spectra showed better performance in predicting IMP content, leading to correlation coefficients $\left(R_{P}\right)$ of 0.951 , root mean square error (RMSEP) of $0.046 \mathrm{mg} / \mathrm{g}$, and residual predictive deviation (RPD) of 3.152. Regression coefficient (RC), successive projections algorithm (SPA), stepwise, competitive adaptive reweighted sampling (CARS), and uninformative variable elimination (UVE) were used to select the optimal wavelengths to optimize the MAS-PLS model. Based on the 18 optimal wavelengths $(907.14,917.02,918.67,926.90,930.20,936.78,956.54,1004.28,1135.89,1211.56$, $1302.07,1367.94,1397.60,1488.31,1680.17,1683.49,1686.80$ and $1695.10 \mathrm{~nm}$ ) selected from MAS spectra by SPA, the MAS-SPA-PLS model was built with $R_{P}$ of 0.920 , RMSEP of $0.056 \mathrm{mg} / \mathrm{g}$ and RPD of 3.220 , which was similar to the MAS-PLS model. The overall study indicated that hyperspectral imaging in the 900-1700 nm range combined with PLS and SPA could be used to predict the IMP content in chicken flesh.
\end{abstract}

Keywords: near-infrared hyperspectral imaging, chicken, inosinic acid, partial least squares, successive projections algorithm DOI: $10.25165 /$ j.ijabe.20221501.6612

Citation: Wang Y Y, He H J, Jiang S Q, Ma H J. Nondestructive determination of IMP content in chilled chicken based on hyperspectral data combined with chemometrics. Int J Agric \& Biol Eng, 2022; 15(1): 277-284.

\section{Introduction}

Compared with pork, beef, and mutton, chicken meat has become one of the most popular products for consumption because of its high protein content, low content of fat, heat energy, and cholesterol $^{[1]}$. With the continuous improvement of people's living standards, the nutrition, flavor, and safety of chicken have become the focus of people's attention ${ }^{[2-4]}$. Umami is considered as the fifth basic taste after sweet, sour, bitter, salty, which not only increases the appetite of consumers but also serves as an important source of nutrition for the human body ${ }^{[5]}$. Studies have found that the umami taste of meat and its products mainly depends on two types of substances, one is amino acids, and the other is nucleotides, and the strongest umami taste is inosinic acid (IMP) ${ }^{[6]}$. IMP, named hypoxanthine nucleotide, the main component of the umami taste of meat, plays an important role in the water holding capacity, physical properties, and sensory properties ${ }^{[7]}$. Umami can be enriched with the accumulation of IMP and other breakdown products in meat, and IMP can produce a unique umami flavor when heated in water or fat ${ }^{[8,9]}$. The ability to increase the umami taste of food using IMP is 40 times stronger than sodium

\section{Received date: 2021-03-22 Accepted date: 2021-09-19}

Biographies: Yangyang Wang, Master, research interest: food rapid and nondestructive detection, Email: yangyang_wang211@126.com; Shengqi Jiang, Master, research interest: food nondestructive analysis and detection, Email: shq jiang@163.com; Hanjun Ma, PhD, Professor, research interest: meat processing and quality control, Email: xxhjma@126.com.

*Corresponding author: Hongju $\mathrm{He}, \mathrm{PhD}$, Professor, research interest: food nondestructive analysis and detection. Henan Institute of Science and Technology, Postdoctoral Research Base, Xinxiang 453003, Henan, China. Tel: +86-373-3040674, +86-13598726097, Fax: +86-373-3040674, Email: hongju_he007@126.com. glutamate $^{[10]}$. Due to the continuous improvement of living standard, consumers have paid more and more attention to pursuing chicken with umami flavor. Compared with other chicken quality parameters, IMP is an important basis for evaluating whether the superior quality or not. IMP has always been regarded as an important index to measure the umami taste of meat in the world, and its content can reflect the pros and cons of the meat flavor. Among the existing methods for the determination of IMP, UV spectrophotometry can only achieve rough quantification, capillary electrophoresis has the problem of low resolution and interference from similar substances such as inosine and adenosine monophosphate, the operation process of thin layer chromatography is complicated and time-consuming, and its recovery rate is low ${ }^{[11,12]}$. Although the high-performance liquid chromatography (HPLC) method is accurate, the sample processing process of this method is cumbersome and difficult ${ }^{[13]}$. In addition, these methods are destructive to samples, time-consuming and laborious, and are not suitable for large-scale and on-site non-destructive IMP content detection of chicken products. New technologies or methods should be developed to meet the increasing requirements for non-destructive determination.

Hyperspectral imaging (HSI) combined spectroscopy and computer vision technology can capture both spectral and spatial information of the tested samples, and has high resolution, non-destructive, easy to operate, and other characteristics ${ }^{[14,15]}$. HSI not only makes up for the shortcomings that traditional spectral technology cannot provide the spatial information of the tested samples, but also breaks the limitation that image techniques cannot provide spectral information ${ }^{[16-18]}$. At present, HSI technology has obtained a great deal of research on nondestructive testing of agricultural products, and has produced many achievements. The technology has been widely used for the 
quality evaluation in livestock meat, such as chemical properties $^{[19-21]}$ (fat, protein, moisture, biogenic amine index, the total volatile basic nitrogen), physical properties ${ }^{[22-25]}$ (color, tenderness and water-holding capacity, $\mathrm{pH}$ ) and microorganism ${ }^{[26]}$.

It has been reported that a variety of acidic components in meats were detected by means of HSI, such as thiobarbituric acid, fatty acid, palmitic acid and oleic acid. Xiong et al. ${ }^{[27]}$ demonstrated that $400-1000 \mathrm{~nm}$ HSI was suitable for the determination of 2-thiobarbituric acid reactive substances (TBARS) values for freshness evaluation in chicken meat with $R_{p}$ of 0.801 and RMSEP of 0.157. There was a similar conclusion in predicting TBARS of pork muscles via HSI in the spectra range of 400-1000 nm, combined with $R_{p}^{2}$ of 0.896 , RMSEP of 1.034 by Aheto et al. ${ }^{[28]}$ Kobayashi et al. researched the contents of total saturated fatty acid (SFA) and total unsaturated fatty acid (UFA) in intact raw beef cuts based on 1000-2300 nm HSI spectra, and the prediction results were good with $R^{2}$ of 0.87 and 0.89 , standard error of prediction (SEP) of $1.69 \%$ and $3.41 \%$, respectively, revealing the applicability and feasibility of $\mathrm{HSI}^{[29]}$. Moreover, the feasibility of combining spectral and textural information from HSI $(900-1700 \mathrm{~nm})$ to improve the prediction of the palmitic acid (C16:0) and oleic acid (C18:1 n9) contents for lamb meat was explored by Wang et al. and $R_{p}{ }^{2}$, RMSEP was 0.9113 and 0.8854 , $0.1368 \mathrm{~g} / 100 \mathrm{~g}$ and $0.3596 \mathrm{~g} / 100 \mathrm{~g}$, respectively ${ }^{[30]}$. However, there were no studies on the determination of IMP in chicken meat by HSI. Given the great potential of HSI in nondestructive detection, we attempted to apply NIR hyperspectral imaging (900-1700 nm) in predicting IMP content in chicken breasts, which will provide the theoretical foundation for further special-purpose equipment development.

\section{Materials and methods}

\subsection{Sample preparation}

One hundred and twenty-seven fresh raw chicken breasts were provided by Zhongpin Food Co., Ltd, China. The length of each chicken breast is $(16.0 \pm 1.0) \mathrm{cm}$ and the width is $(10.0 \pm 1.0) \mathrm{cm}$. All samples were vacuum packed, frozen and transported for around $3 \mathrm{~h}$ (maintain IMP level to the maximum extent) to the Meat Processing and Quality Control Laboratory of Henan Institute of Science and Technology, Xinxiang, China. Each fresh raw chicken breast was cut into $3 \mathrm{~cm}$ length $\times 3 \mathrm{~cm}$ width $\times 1 \mathrm{~cm}$ thickness squares under aseptic conditions, and finally, 127 samples were obtained. All the samples were put into sterile disposable plastic boxes, labeled and stored in a digital refrigerator at $0^{\circ} \mathrm{C}-4^{\circ} \mathrm{C}$ for $0,1,2,3,4,5$, and $6 \mathrm{~d}$ (slight decrease of IMP). Eighteen samples at first six days and nineteen samples at last day were taken out from the refrigerator to determine IMP.

\subsection{Hyperspectral image acquisition and calibration}

In this experiment, an advanced NIR hyperspectral reflectance imaging system in the range of 900-1700 nm (HSI-eNIR-XC130, Isuzu Optics Corp., Taiwan) was used to collect the spectral information of chicken samples. The system is mainly composed of a spectrograph, a Charged Couple Device (CCD) camera, zoom lens, illumination source, a moving stage, and a computer equipped with Spectral Image software and HSI Analyzer software, as shown in Figure 1.

The HSI system was turned on and preheated for $30 \mathrm{~min}$ in order to stabilize the light source and reduce the error caused by the instability of the spectral system. The sample was taken from the refrigerator to reach room temperature, and then put on the moving table of the system for scanning. The parameter settings of the hyperspectral system are listed in Table 1 . The dark current of the camera will cause a lot of noise in the sample image acquisition process. The original images need to be corrected with black and white images to reduce the influence of noise on the hyperspectral data. The calibration can be calculated using Equation (1) ${ }^{[31]}$

$$
R=\frac{R_{0}-R_{B}}{R_{W}-R_{B}}
$$

where, $R$ is the calibrated reflectance image; $R_{0}$ is the raw image; $R_{B}$ is the black image ( $0 \%$ reflectance) and $R_{W}$ is the white image ( $99.9 \%$ reflectance). $\quad R_{W}$ was obtained by recording an image of a white board; $R_{B}$ was achieved by covering the lens with cap and then collecting an image after turning off the light source completely.

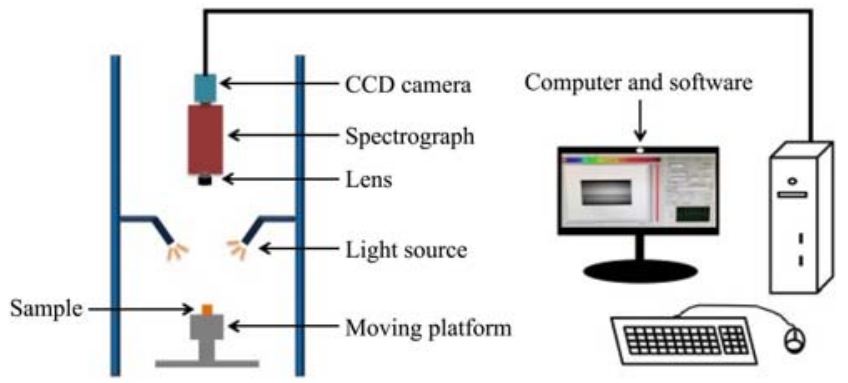

Figure 1 Schematic diagrams of main components of the HSI systems

Table 1 Parameters setting of HSI system

\begin{tabular}{lc}
\hline \multicolumn{1}{c}{ Parameter setting } & Parameter \\
\hline Exposure time & $3.6 \mathrm{~ms}$ \\
Wavelength range & $900-1700 \mathrm{~nm}$ \\
Speed of scanning & $6.54 \mathrm{~mm} / \mathrm{s}$ \\
Scanning distance & $65-165 \mathrm{~mm}$ \\
Image size & $640 \times 848 \mathrm{dpi}$ \\
Object distance & $44 \mathrm{~cm}$ \\
Number of wavelengths & 486 \\
\hline
\end{tabular}

\subsection{Spectral data extraction}

After the image calibration, the image corresponding to the location of the chicken sample was considered as the region of interest $(\mathrm{ROI})^{[32]}$. The spectra within the ROI were extracted and averaged by HSI Analyzer software to achieve mean spectral data of each sample, which is a critical step in data processing to remove the undesired interference such as the background and sample platform. By repeating the identification and extraction process, 127 mean spectra corresponding to the 127 samples were obtained. The mean spectra of all samples were saved in a matrix where the rows represented the 127 samples and the columns represented the 486 wavelengths as the spectroscopic resolution of HSI was about $1.6 \mathrm{~nm}$ in the range of $900-1700 \mathrm{~nm}$.

\subsection{Determination of IMP}

\subsubsection{Sample pretreatment}

After the chicken sample was minced with a meat grinder, about $5 \mathrm{~g}$ of the samples were weighed. $20 \mathrm{~mL}$ perchloric acid of $6 \%$ and the chicken samples weighed were added to the $50 \mathrm{~mL}$ centrifuge tube and homogenized until well mixed. After the homogenate was centrifuged at $8000 \mathrm{r} / \mathrm{min}$ for $15 \mathrm{~min}$, the supernatant was filtered into a $100 \mathrm{ml}$ triangulated bottle. The precipitate was homogenized again with $15 \mathrm{~mL} 6 \%$ perchloric acid and centrifuged to obtain the second supernatant. The twice-acquired supernatants were combined, adjusted to $\mathrm{pH} 6.5$ using potassium hydroxide solution, transferred to a $100 \mathrm{~mL}$ volumetric flask and mixed evenly. The mixed supernatants were filtered with a $0.45 \mu \mathrm{m}$ filter membrane and then used for HPLC analysis. 


\subsubsection{Chromatographic condition}

In the HPLC analysis, the chromatographic column was C18-ST $(5 \mu \mathrm{m}, 120 \mathrm{~A}, 4.6 \mathrm{~mm} \times 250 \mathrm{~mm}$, Beijing TechMate Technology Corp., China). The mobile phase was $20 \%$ methanol solution with a flow velocity of $1 \mathrm{~mL} / \mathrm{min}$. The injection volume was $5 \mu \mathrm{L}$. The ultraviolet detection wavelength of refractive index detector (2414, Waters Corp., the United States) was $254 \mathrm{~nm}$. The chromatographic temperature was $25.0^{\circ} \mathrm{C}$. Both the sample extracting solution and the standard substance solution run for $10 \mathrm{~min}$ in the HPLC system (e2695, Waters Corp., the United States). The chromatographic conditions for IMP detection were improved on the basis of the research of Zhang et al. ${ }^{[7]}$. The chromatograms of the standard solution and the sample extraction solution were shown in Figure 2.
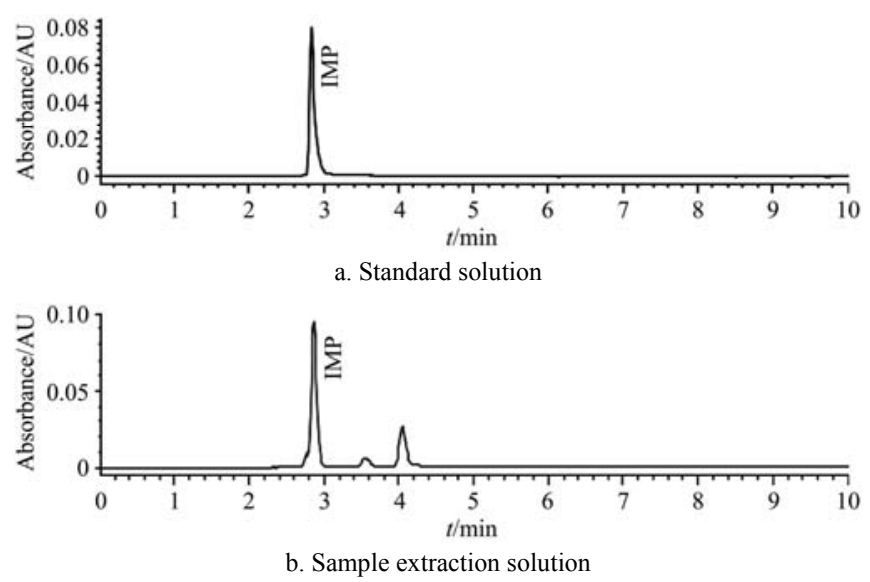

Figure 2 Chromatograms of the standard solution and the sample extraction solution of IMP

\subsubsection{Determination of regression equation of standard curve}

The IMP of $0.02,0.04,0.08,0.10,0.12$ and $0.16 \mathrm{mg} / \mathrm{mL}$ standard working liquid were prepared from $1 \mathrm{mg} / \mathrm{mL}$ IMP standard solution, respectively, and analyzed using Section 2.4.2 chromatographic conditions. The concentration of IMP standard was taken as independent variable $X$, while the response value of chromatographic peak area was taken as dependent variable $Y$. The results showed that there was a good linear relationship between IMP concentration and peak area response between $0.02-0.16 \mathrm{mg} / \mathrm{mL}$. The calculated standard curve equation of IMP was $Y=4.81 \times 10^{6} X-2.58 \times 10^{4}$ with the correlation coefficient of 0.9997. The standard curve was shown in Figure 3.

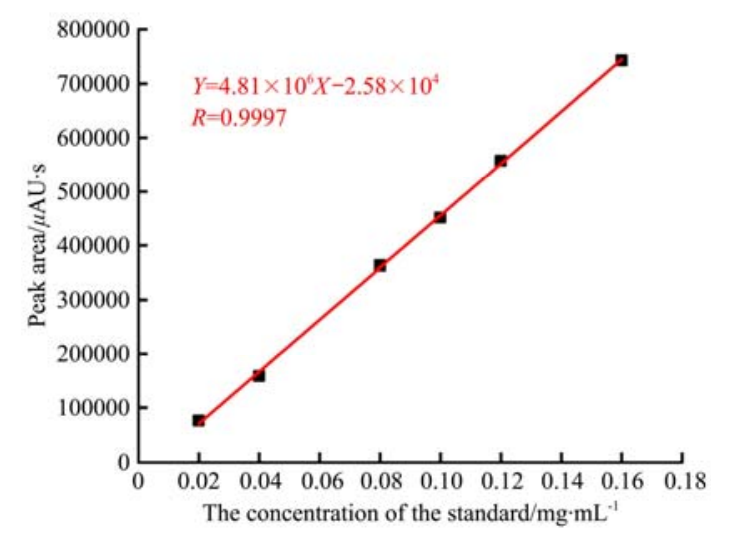

Figure 3 Response values of chromatographic peak area of IMP standard concentration

2.4.4 Calculation of IMP content in samples

According to Section 2.4.3 chromatographic conditions, the sample extraction solution was analyzed, the content of IMP ( $\mathrm{mg} / \mathrm{g})$ in the sample was calculated by Equation (2).

$$
X=\frac{100 C}{m}
$$

where, $X$ is the content of IMP in the sample, $\mathrm{mg} / \mathrm{g} ; C$ is the concentration of IMP in the sample extraction solution, $\mathrm{mg} / \mathrm{mL} ; m$ is the weight of the sample, $g$.

\subsection{Spectral data pre-processing}

In the process of spectral acquisition, the spectra may be interfered with by noise interference generated by the instrument itself and the external environment, and the influence of light scattering. Therefore, it is necessary to perform spectral preprocessing to eliminate negative effects. This study used eight commonly used preprocessing methods to preprocess the original spectra of the sample, including moving average smoothing (MAS), Gaussian filter smoothing (GFS), Savitzky-Golay smoothing (SGS), normalize (NOR), multiplicative scatter correction (MSC), standard normal variate (SNV), Gap-Segment derivatives (GSD) spectra and Norris gap derivatives (NGD). MAS, GFS, and SGS are smoothing processing to remove random noise ${ }^{[20,33]}$. NOR can eliminate the effect of optical path change or sample dilution on the spectrum ${ }^{[34]}$. MSC and SNV are mainly used for light scattering correction to improve the accuracy of spectral data ${ }^{[35,36]}$. Both GSD and NGD belong to derivatives that have the capability to remove both additive and multiplicative effects in the spectra and have been used in analytical spectroscopy for many years ${ }^{[37]}$.

\subsection{Establishment of IMP prediction model}

Partial least squares (PLS) is a high-efficiency multivariate data analysis method that combines the functions of multiple linear regression (MLR) and principal component regression (PCR), and has a wide range of applications and predictability ${ }^{[38,39]}$. In this experiment, PLS was used to mine the quantitative relationship between the spectral data and IMP content of chicken samples. The reflectance spectra of $900-1700 \mathrm{~nm}$ were used as the independent variable, the reference value of IMP was used as the dependent variable, PLS prediction model was established with the two variables to predict IMP content in chicken.

The performance of the PLS models was assessed by correlation coefficient $(R)$ of calibration set $\left(R_{C}\right)$ and prediction set $\left(R_{P}\right)$ and root mean square error (RMSE) of calibration set (RMSEC) and prediction set (RMSEP). In general, the closer $R$-value to 1 and the smaller RMSE value represent the model is more stable and predictable. Robustness and residual prediction deviation (RPD) can also be used to evaluate the performance of the model. Robustness is expressed by $\mid$ RMSEC-RMSEP $\mid(\Delta E)$, the smaller the $\Delta E$ value, the more stable the model ${ }^{[24]}$. RPD is the ratio of the standard deviation of the reference value of all samples in the prediction set to the standard deviation of the prediction set ${ }^{[40]}$. The higher $R$ and RPD values and the smaller RMSE and $\Delta E$ values indicate that the PLS model has better predictive ability and stronger stability. The above evaluation parameters are calculated according to Equations (3)-(7).

$$
\begin{gathered}
R=\frac{\sum_{i=1}^{n}\left(x_{i}-\bar{x}\right)\left(y_{i}-\bar{y}\right)}{\sqrt{\sum_{i=1}^{n}\left(x_{i}-\bar{x}\right)^{2}} \sqrt{\sum_{i=1}^{n}\left(y_{i}-\bar{y}\right)^{2}}} \\
\mathrm{RMSE}=\sqrt{\frac{1}{n} \sum_{i=1}^{n}\left(x_{i}-y_{i}\right)^{2}} \\
\mathrm{SEP}=\sqrt{\frac{\sum_{i=1}^{n}\left(\bar{y}-y_{i}-\mathrm{Bias}\right)^{2}}{n-k-1}} \\
\Delta E=\mid \text { RMSEC }-\mathrm{RMSEP} \mid
\end{gathered}
$$




$$
\mathrm{RPD}=\frac{\mathrm{SD}}{\mathrm{SEP}}
$$

where, $x_{i}$ is the actual measured IMP content of the sample, i.e., $i=1$, $2,3, \ldots, n$ and $n$ is the total number of samples; $\bar{x}$ is the average value of $x_{i}, y_{i}$ is the predictive value of the IMP content of the sample; $\bar{y}$ is the average value of $y_{i}$; SEP is the prediction standard deviation of the prediction set; Bias is the systematic deviation; $k$ is the number of regression factors; SD is the standard deviation of IMP content of all samples in the prediction set.

\subsection{Optimal wavelength selection and model optimization}

There were a total of 486 wavelengths in the $900-1700 \mathrm{~nm}$ range used in this experiment. Using all wavelengths as input variables for modeling can achieve a good prediction effect, but a large amount of data in the whole band contains more redundant information which will reduce the efficiency of model performance and affect the predictive ability of the model. Although the use of different preprocessing methods to process the original data can remove the negative effects of the system, the preprocessing cannot eliminate redundant information and retain useful information that contributes to the IMP content model. Therefore, it is necessary to select optimal wavelengths for modeling by eliminating irrelevant information and keeping useful information.
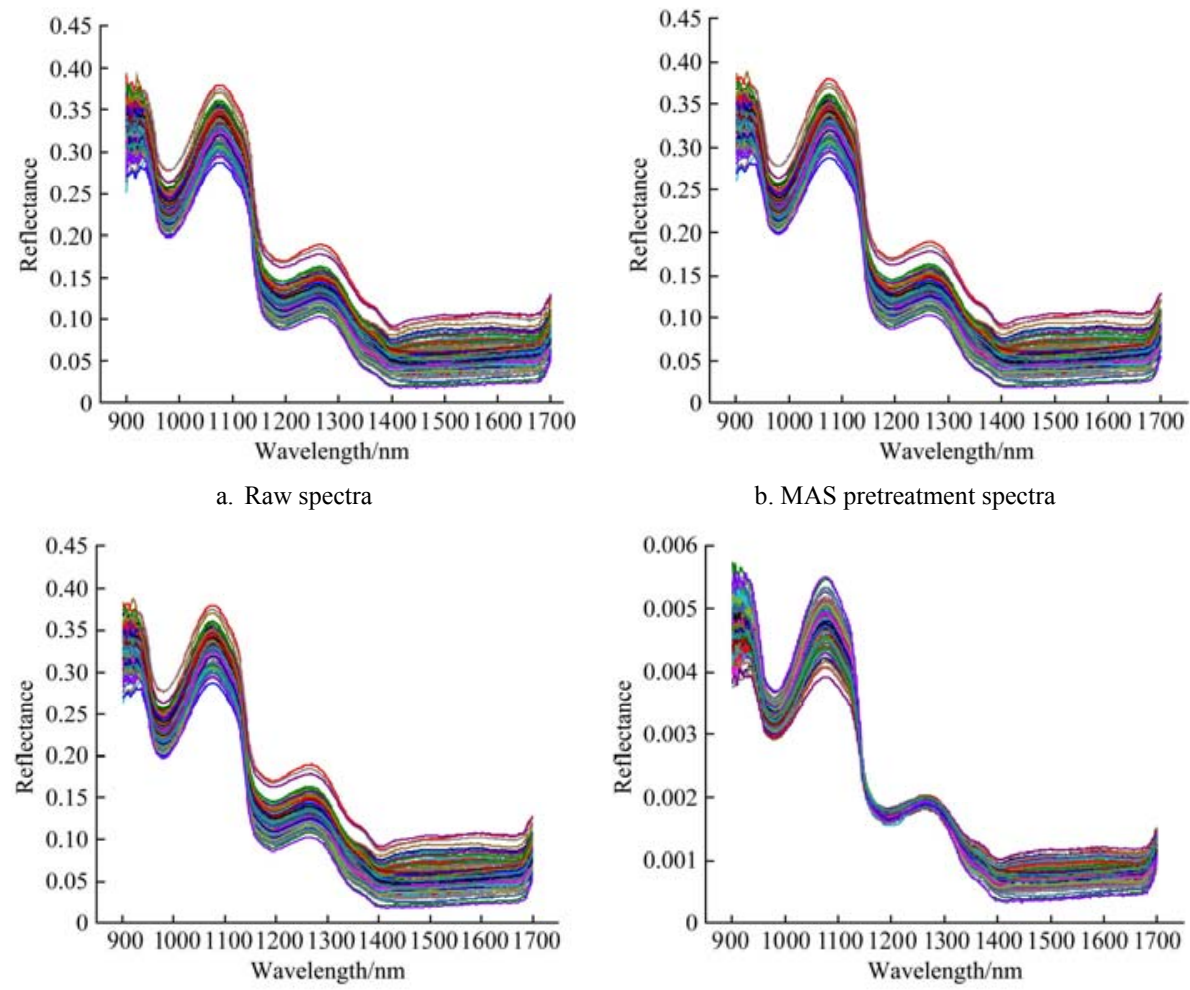

d. SGS pretreatment spectra

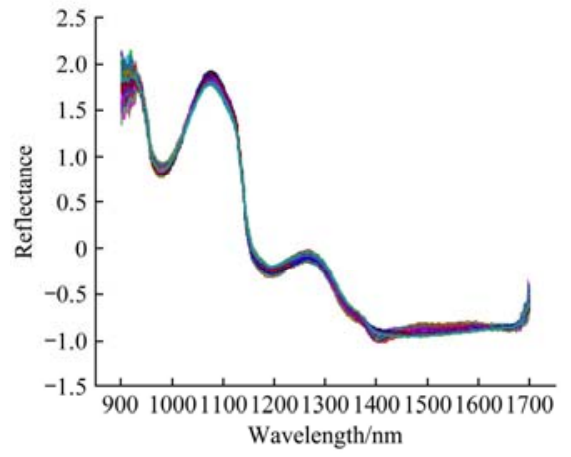

g. SNV pretreatment spectra

b. MAS pretreatment spectra
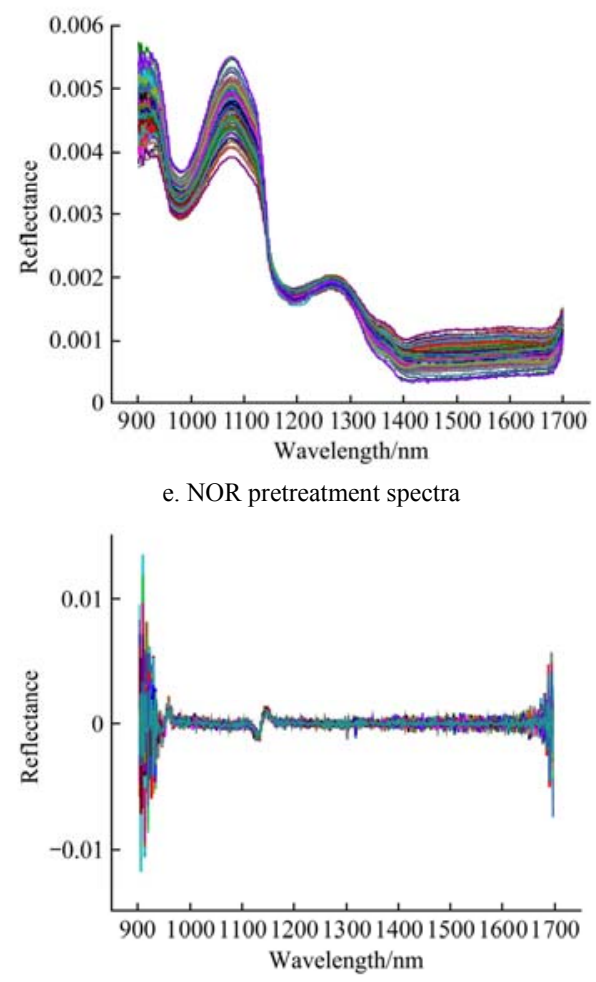

h. GSD pretreatment spectra
The regression coefficient method $(\mathrm{RC})^{[41]}$, stepwise ${ }^{[42]}$, successive projection algorithm (SPA) ${ }^{[43]}$, competitive adaptive reweighted sampling (CARS $)^{[44],}$ and uninformative variable elimination (UVE) ${ }^{[45]}$ were used to select the optimal wavelengths. These five methods are widely used in the selection of spectral model variables, which can achieve variable dimensionality reduction during the modeling process, thereby reducing the singularity and instability of the original spectral data. By comparing the performance of the optimized model, the best method for selecting the optimal wavelengths was confirmed. The wavelength selection by RC method and the PLS model construction was implemented by using software Unscrambler 9.7 (CAMO, Oslo, Norway). The Stepwise, SPA, and CARS processes were implemented by Matlab R2016a software (The Mathworks, Inc., Natick, MA, USA).

\section{Results and discussion}

\subsection{Spectral profiles of chicken breast}

As can be seen from Figure 4, although the trends of all the spectral curves of 127 chicken samples were similar for raw spectra, the high and low positions were different, which is probably due to the different chemical composition content of the 127 chicken
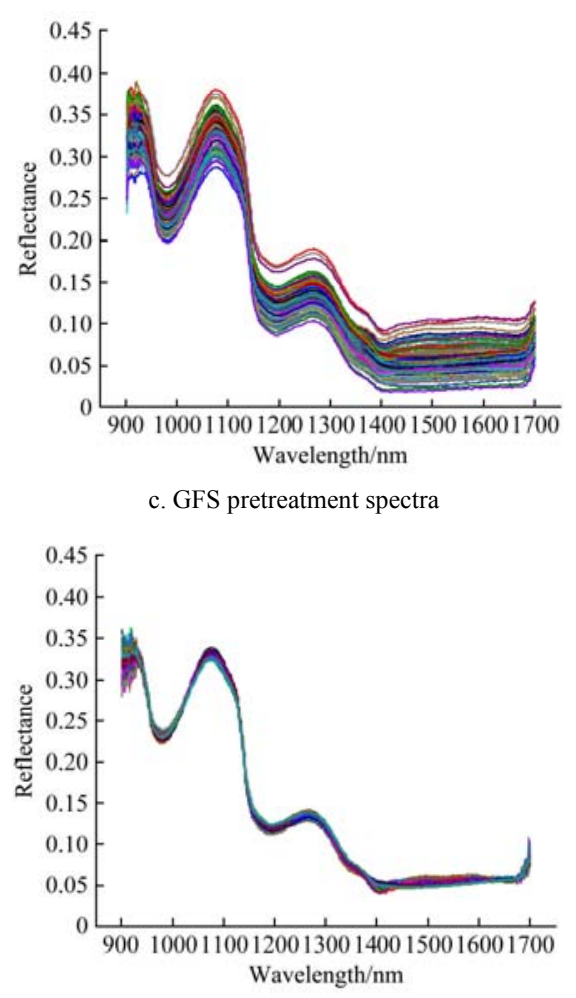

f. MSC pretreatment spectra

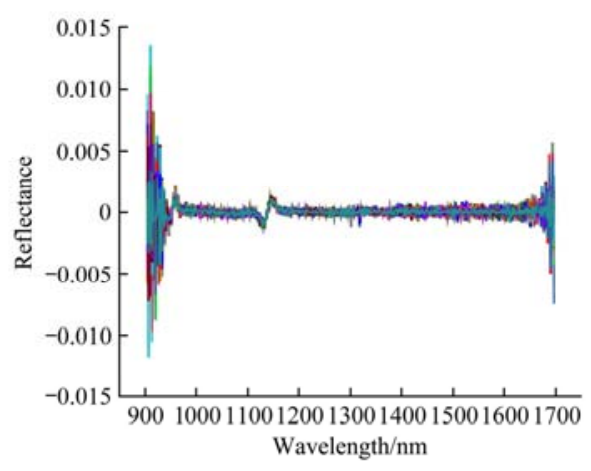

i. NGD pretreatment spectra

Figure 4 NIR characteristics of chicken samples under different pretreatment 
samples corresponding to diverse spectral characteristics. In specific, the absorption peak at around $980 \mathrm{~nm}$ and $1450 \mathrm{~nm}$ are related to water content (O-H stretching vibration), while the peak around $1200 \mathrm{~nm}$ is attributed to fat $(\mathrm{C}-\mathrm{H} \text { stretching vibration })^{[46]}$. No typical protein absorption was found as it was masked by water absorption, resulting from the more than $70 \%$ water content in chicken flesh. Although no absorption peak was found related to IMP, appropriate chemometric algorithms could be adopted to mine the spectral information from the full $900-1700 \mathrm{~nm}$ range to relate to IMP. After different pretreatments, the original spectrum curves had different changes in shape, which indicated the different noise elimination effects.

\subsection{Reference IMP values}

The reference IMP values of the 127 chicken samples measured by HPLC were obtained and shown in Table 2. All the values were then sorted from smallest to largest, and one in four was taken into prediction set and the remaining was put into calibration $\operatorname{set}^{[47]}$. Figure 5 vividly illustrated that the reference IMP values of 127 chicken samples obeyed the normal distribution, which demonstrated it was reasonable to establish a prediction model for chicken IMP.

Table 2 Reference values of IMP (mg/g) measured by HPLC method

\begin{tabular}{cccccc}
\hline Sample set & $\begin{array}{c}\text { Numer of } \\
\text { samples }\end{array}$ & $\begin{array}{c}\text { Minimum } \\
/ \mathrm{mg} \cdot \mathrm{g}^{-1}\end{array}$ & $\begin{array}{c}\text { Maximum } \\
/ \mathrm{mg} \cdot \mathrm{g}^{-1}\end{array}$ & $\begin{array}{c}\text { Mean } \\
/ \mathrm{mg} \cdot \mathrm{g}^{-1}\end{array}$ & $\begin{array}{c}\mathrm{SD} \\
/ \mathrm{mg} \cdot \mathrm{g}^{-1}\end{array}$ \\
\hline Calibration set & 95 & 0.710 & 1.479 & 0.989 & 0.138 \\
Prediction set & 32 & 0.767 & 1.429 & 0.992 & 0.145 \\
\hline
\end{tabular}

Note: SD: Standard Deviation.

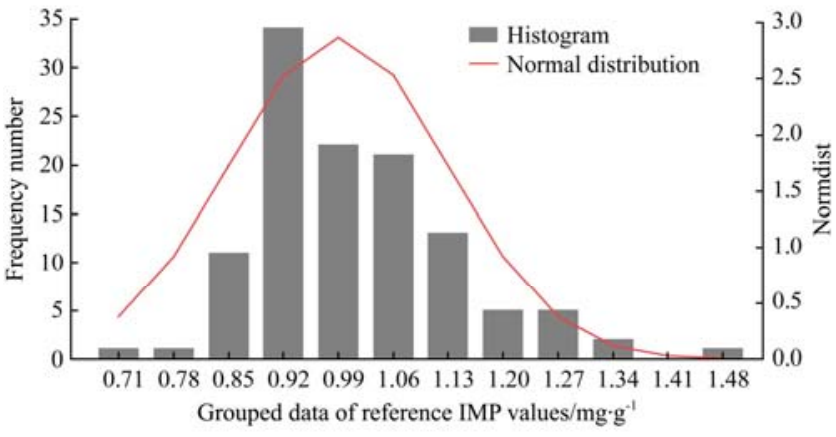

Figure 5 Normal distribution of reference IMP values

3.3 PLS model for predicting IMP values using full wavelength

PLS algorithm was implemented by inputting a matrix with 127 samples $\times 486$ wavelengths to find the quantitative relationship between spectra and reference IMP values. Among, the sub-matrix of 95 samples $\times 486$ wavelengths was used for calibration and the rest sub-matrix of 32 samples $\times 486$ wavelengths was for prediction. With Raw, MAS, GFS, SGS, NOR, MSC, SNV, GSD and NGD spectra, nine full wavelengths PLS models were established and their performances are listed in Table 3.

It can be seen that different pretreatment methods had different effects on the PLS models for predicting IMP content in chicken. Among, the $R_{C}$ and $R_{P}$ of the Raw-PLS, MAS-PLS, GFS-PLS and SGS-PLS models were all reached over 0.940, which indicated the good linear correlation between spectra and IMP values. By comparison, the $R_{C}$ and $R_{P}$ values of the MAS-PLS model were the highest (0.964 and 0.951, respectively), the RMSEC, RMSEP and $\Delta E$ values were the smallest $(0.036 \mathrm{mg} / \mathrm{g}, 0.046 \mathrm{mg} / \mathrm{g}$, and $0.010 \mathrm{mg} / \mathrm{g}$, respectively), indicating the highest accuracy and stability. Besides, the highest RPD value of 3.152 represented that the MAS-PLS model was moderately successful and therefore considered for the further test with the best performance.

3.4 Optimal wavelength selected by the chemometric method

Although the predictive ability of the MAS-PLS model for IMP prediction was good, a total of 486 wavelengths in the 900-1700 nm range would require more time during the modeling, which would reduce the efficiency of hyperspectral detection. Therefore, the number of full wavelengths should be decreased and the optimal wavelengths should be selected to achieve the optimization of MAS-PLS model. In view of this, RC, stepwise, SPA, CARS and UVE were used to select the optimal wavelengths and the results are listed in Table 4. The 18-31 optimal wavelengths were selected from the 486 full wavelengths, indicating that $92.80 \%-96.30 \%$ wavelengths were reduced. Within the five methods, the SPA method selected the 18 optimal wavelengths with the least number of wavelengths and had the highest wavelength reduction rate.

\subsection{PLS model for predicting IMP values using optimal wavelengths}

Based on the above selected optimal wavelengths, five optimized PLS models were respectively established, and their performances in predicting IMP content were shown in Table 5. With higher $R$, RPD and lower RMSE and $\triangle \mathrm{E}$, the MAS-SPA-PLS model established with 18 optimal wavelengths selected by SPA showed better accuracy and stability in predicting IMP content, resulting in $R_{P}$ of 0.920 , RMSEP of $0.056 \mathrm{mg} / \mathrm{g}, \Delta E$ of 0.013 and RPD of 3.220. Besides, the MAS-SPA-PLS model showed similar performance compared with the MAS-PLS model $\left(R_{P}\right.$ of 0.951 , RMSEP of $0.046 \mathrm{mg} / \mathrm{g}, \Delta E$ of 0.010 and RPD of 3.152). Hence, the MAS-SPA-PLS model was more suitable to predict IMP content in chicken meat with higher efficiency.

Table 3 PLS models for predicting IMP by using the full range spectra

\begin{tabular}{|c|c|c|c|c|c|c|c|c|}
\hline \multirow{2}{*}{$\begin{array}{c}\text { Spectral } \\
\text { preprocessing }\end{array}$} & \multirow{2}{*}{ Model } & \multirow{2}{*}{$\begin{array}{c}\text { Number of } \\
\text { wavelengths }\end{array}$} & \multicolumn{2}{|c|}{ Calibration set } & \multicolumn{2}{|c|}{ Prediction set } & \multirow{2}{*}{$\Delta E$} & \multirow{2}{*}{ RPD } \\
\hline & & & $R_{C}$ & $\mathrm{RMSEC} / \mathrm{mg} \cdot \mathrm{g}^{-1}$ & $R_{P}$ & $\mathrm{RMSEP} / \mathrm{mg} \cdot \mathrm{g}^{-1}$ & & \\
\hline Raw & Raw-PLS & 486 & 0.959 & 0.039 & 0.948 & 0.047 & 0.011 & 3.021 \\
\hline MAS & MAS-PLS & 486 & 0.964 & 0.036 & 0.951 & 0.046 & 0.010 & 3.152 \\
\hline GFS & GFS-PLS & 486 & 0.958 & 0.039 & 0.941 & 0.051 & 0.012 & 2.959 \\
\hline SGS & SGS-PLS & 486 & 0.960 & 0.038 & 0.940 & 0.051 & 0.013 & 2.900 \\
\hline NOR & NOR-PLS & 486 & 0.927 & 0.051 & 0.871 & 0.070 & 0.019 & 2.042 \\
\hline MSC & MSC-PLS & 486 & 0.888 & 0.063 & 0.849 & 0.076 & 0.013 & 1.908 \\
\hline SNV & SNV-PLS & 486 & 0.888 & 0.063 & 0.849 & 0.076 & 0.013 & 1.883 \\
\hline GSD & GSD-PLS & 486 & 0.950 & 0.043 & 0.753 & 0.098 & 0.055 & 1.510 \\
\hline NGD & NGD-PLS & 486 & 0.950 & 0.043 & 0.753 & 0.098 & 0.055 & 1.510 \\
\hline
\end{tabular}

Note: $R_{C}$ : Regression Coefficient; $R_{P}$ : Correlation Coefficients; RMSEP: Root Mean Square Error; RPD: Residual Predictive Deviation; RMSEC: Root Mean Square Error of Calibration. $\quad \Delta E=\mid$ RMSEC-RMSEP|. 
Table 4 Results of optimal wavelengths selected by different methods for IMP content prediction

\begin{tabular}{|c|c|c|c|c|c|}
\hline Spectra & $\begin{array}{l}\text { Number of full } \\
\text { wavelength }\end{array}$ & Method & $\begin{array}{l}\text { The specific } \\
\text { optimal wavelengths/nm }\end{array}$ & $\begin{array}{l}\text { Number of optimal } \\
\text { wavelengths }\end{array}$ & $\begin{array}{l}\text { Wavelength } \\
\text { reduction }\end{array}$ \\
\hline \multirow{5}{*}{ MAS } & \multirow{5}{*}{486} & $\mathrm{RC}$ & $\begin{array}{l}907.14,918.67,921.96,926.90,930.20,935.14,1020.73,1027.31,1132.60,1165.50, \\
1302.07,1339.94,1372.89,1531.24,1554.37,1680.17,1683.49,1688.46,1691.78, \\
1696.76\end{array}$ & 20 & $95.88 \%$ \\
\hline & & Stepwise & $\begin{array}{l}907.14,925.26,931.84,956.54,958.19,969.71,971.36,994.40,1134.25,1341.59, \\
1343.24,1480.05,1522.98,1531.24,1541.15,1542.80,1551.06,1589.09,1590.75, \\
1685.15,1693.44,1695.10\end{array}$ & 22 & $95.47 \%$ \\
\hline & & SPA & $\begin{array}{l}907.14,917.02,918.67,926.90,930.20,936.78,956.54,1004.28,1135.89,1211.56, \\
1302.07,1367.94,1397.60,1488.31,1680.17,1683.49,1686.80,1695.10\end{array}$ & 18 & $96.30 \%$ \\
\hline & & CARS & $\begin{array}{l}907.14,912.08,915.38,918.67,925.26,926.90,930.20,951.60,954.90,956.54,958.19, \\
1025.67,1028.96,1030.61,1037.19,1043.77,1129.31,1130.96,1132.60,1134.25, \\
1216.50,1303.72,1307.01,1313.60,1318.54,1330.06,1389.36,1405.84,1552.72, \\
1554.37,1556.02,1589.09,1683.49,1691.78,1696.76\end{array}$ & 35 & $92.80 \%$ \\
\hline & & UVE & $\begin{array}{l}907.14,908.79,915.38,920.32,925.26,956.54,968.07,969.71,1027.31,1028.96, \\
1030.61,1043.77,1045.41,1102.99,1144.12,1267.51,1298.78,1300.43,1302.07, \\
1348.18,1349.82,1374.53,1419.03,1488.31,1494.91,1511.42,1552.72,1554.37, \\
1556.02,1557.68,1579.17\end{array}$ & 31 & $93.62 \%$ \\
\hline
\end{tabular}

Table 5 PLS models for predicting IMP by using the optimal wavelengths

\begin{tabular}{|c|c|c|c|c|c|c|c|c|}
\hline \multirow{2}{*}{ Model } & \multirow{2}{*}{ Method } & \multirow{2}{*}{ Number of wavelengths } & \multicolumn{2}{|c|}{ Calibration set } & \multicolumn{2}{|c|}{ Prediction set } & \multirow{2}{*}{$\Delta E$} & \multirow{2}{*}{ RPD } \\
\hline & & & $R_{C}$ & $\mathrm{RMSEC} / \mathrm{mg} \cdot \mathrm{g}^{-1}$ & $R_{P}$ & $\mathrm{RMSEP} / \mathrm{mg} \cdot \mathrm{g}^{-1}$ & & \\
\hline MAS-RC-PLS & $\mathrm{RC}$ & 20 & 0.944 & 0.045 & 0.873 & 0.071 & 0.027 & 2.013 \\
\hline MAS-Stepwise-PLS & Stepwise & 22 & 0.945 & 0.035 & 0.895 & 0.065 & 0.031 & 2.196 \\
\hline MAS-SPA-PLS & SPA & 18 & 0.950 & 0.043 & 0.920 & 0.056 & 0.013 & 3.220 \\
\hline MAS-CARS-PLS & CARS & 35 & 0.942 & 0.046 & 0.890 & 0.066 & 0.021 & 2.163 \\
\hline MAS-UVE-PLS & UVE & 31 & 0.860 & 0.059 & 0.860 & 0.074 & 0.002 & 1.932 \\
\hline
\end{tabular}

The $F$-test on IMP values in the MAS-SPA-PLS model was conducted for the sake of statistical soundness ${ }^{[48]}$. As shown in Table 6 , the $F<" F$ (one-tailed critical value)" and $P(F \leq f)>0.05$ of MAS-SPA-PLS model predicting results, that was to say $1.18<1.82$ and $0.32>0.05$, which indicated there was no significant difference in precision between the two groups of data (reference value and predicted value). It was appropriate for the MAS-SPA-PLS model to detect IMP in chicken breast by HSI and the scatter plot of measured and predicted values in the prediction set are shown in Figure 6. The prediction model can be expressed as Equation (8).

$$
\begin{aligned}
Y_{M A S-S P A-P L S}= & 2.18-10.47 X_{907.14 \mathrm{~nm}}-8.48 X_{917.02 \mathrm{~nm}}+12.10 X_{918.67 \mathrm{~nm}} \\
& +15.63 X_{926.90 \mathrm{~nm}}-11.15 X_{930.20 \mathrm{~nm}}+10.95 X_{936.78 \mathrm{~nm}} \\
& -32.47 X_{956.54 \mathrm{~nm}}+21.09 X_{1004.28 \mathrm{~nm}}-15.25 X_{1135.89 \mathrm{~nm}} \\
& +10.28 X_{1211.56 \mathrm{~nm}}+18.36 X_{1302.07 \mathrm{~nm}}-0.89 X_{1367.94 \mathrm{~nm}} \\
& +18.49 X_{1397.60 \mathrm{~nm}}-16.75 X_{1488.31 \mathrm{~nm}}-7.42 X_{1680.17 \mathrm{~nm}} \\
& +18.64 X_{1683.49 \mathrm{~nm}}-12.44 X_{1686.80 \mathrm{~nm}}-7.89 X_{1695.10 \mathrm{~nm}}
\end{aligned}
$$

Table 6 F-test two-sample analysis of variance of optimized model

\begin{tabular}{lccc}
\hline Optimization model & Items & Reference value & Predicted value \\
\hline & Average & 0.99 & 0.99 \\
& Variance & 0.02 & 0.02 \\
& Observed value & 32 & 32 \\
MAS-SPA-PLS & $\mathrm{df}$ & 31 & 31 \\
& $F$ & 1.18 & - \\
& $P(F \leq f)$ & 0.32 & - \\
& $\begin{array}{c}F \text { (one-tailed } \\
\text { critical value })\end{array}$ & 1.82 & - \\
\hline
\end{tabular}

Note: df: degree of freedom.

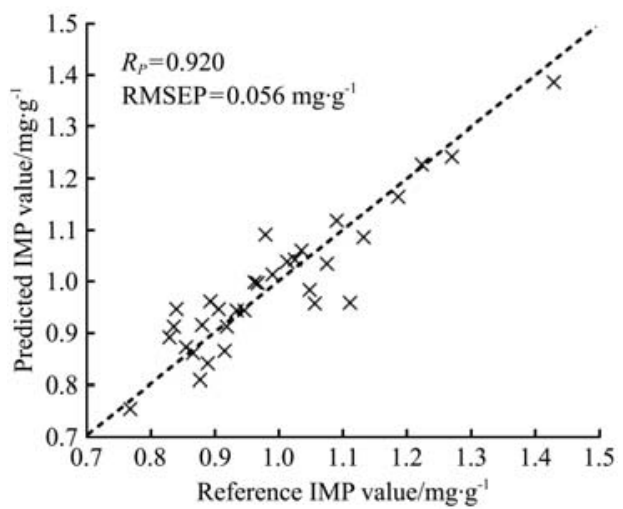

Figure 6 Scatter plot of measured and predicted values of MAS-SPA-PLS model

\section{Conclusions}

In this experiment, the potential of 900-1700 nm high-resolution HSI spectra were investigated for the non-destructive prediction of IMP content in chicken breast. PLS algorithm was applied to mine the linear relationship between Raw, MAS, GFS, SGS, NOR, MSC, SNV, GSD, NGD spectra and IMP values, respectively. The results showed that the MAS-PLS model performed better for predicting IMP content with $R_{P}$ of 0.951 and RMSEP of $0.046 \mathrm{mg} / \mathrm{g}$. After simplifying 486 full wavelengths of MAS spectra by SPA, the MAS-SPA-PLS model built with 18 optimal wavelengths was better in predicting IMP content, with $R_{P}$ of 0.920 and RMSEP of $0.056 \mathrm{mg} / \mathrm{g}$. In conclusion, it is promising to predict IMP content in chicken breast based on HSI in a non-destructive way. 


\section{Acknowledgements}

The authors acknowledge that this work was financially supported by the Major Scientific and Technological Project of Henan Province (Grant No. 182102310060; No. 161100110600), Key Scientific and Technological Project of Henan Province (Grant No. 212102310491), China Postdoctoral Science Foundation (Grant 2018M632767), Henan Postdoctoral Science Foundation (Grant No. 001801021), and Youth Talents Lifting Project of Henan Province (Grant No. 2018HYTP008).

\section{[References]}

[1] Mellen M, Pavelková A, Haščík P, Bobko M, Čuboň J. Sensory evaluation of coob 500 chicken meat after application of different additives in their nutrition. Potravinarstvo, 2014; 8(1): 184-189.

[2] Grau R, Sanchez A J, Giron J, Iborra E, Fuentes A, Barat J M. Nondestructive assessment of freshness in packaged sliced chicken breasts using SW-NIR spectroscopy. Food Research International, 2011; 44(1): 331-337.

[3] Jayasena D D, Ahn D U, Nam K C, Jo C. Flavour chemistry of chicken meat: A review. Asian-Australasian Journal of Animal Sciences, 2013; 26(5): 732-742.

[4] Khulal U, Zhao J, Hu W, Chen Q. Intelligent evaluation of total volatile basic nitrogen (TVB-N) content in chicken meat by an improved multiple level data fusion model. Sensors and Actuators B: Chemical, 2017; 238: 337-345.

[5] Zhao Y, Zhang M, Devahastin S, Liu Y. Progresses on processing methods of umami substances: A review. Trends in Food Science \& Technology, 2019; 93: 125-135.

[6] Iwamoto E, Oka A, Iwaki F. Effects of the fattening period on the fatty acid composition of fat deposits and free amino acid and inosinic acid contents of the longissimus muscle in carcasses of Japanese Black steers. Animal Science Journal, 2009; 80(4): 411-417.

[7] Zhang G Q, Ma Q G, Ji C. Effects of dietary inosinic acid on carcass characteristics, meat quality, and deposition of inosinic acid in broilers. Poultry Science, 2008; 87(7): 1364-1369.

[8] Dashdorj D, Amna T, Hwang I. Influence of specific taste-active components on meat flavor as affected by intrinsic and extrinsic factors: an overview. European Food Research \& Technology, 2015; 241(2): 157-171.

[9] Clausen M P, Christensen M, Djurhuus T H, Duelund L, Mouristsen O G. The quest for umami: can sous vide contribute? International Journal of Gastronomy \& Food Science, 2018; 13: 129-133.

[10] Wang G, Sun J, Yao Y, An X, Zhang H, Chu G, et al. Detection of Inosine Monophosphate (IMP) in meat using double-enzyme sensor. Food Analytical Methods, 2020; 13(5): 420-432.

[11] Rikimaru K, Takahashi H. Evaluation of the meat from Hinai-jidori chickens and broilers: analysis of general biochemical components, free amino acids, inosine 5'-monophosphate, and fatty acids. Journal of Applied Poultry Research, 2010; 19(4): 327-333.

[12] Wang X F, Liu G H, Cai H Y, Chang W H, Ma J S, Zheng A J, et al. Attempts to increase inosinic acid in broiler meat by using feed additives. Poultry Science, 2014; 93(11): 2802-2808.

[13] Utama D T, Baek K H, Jeong H S, Yoon S K, Lee S K. Effects of cooking method and final core-temperature on cooking loss, lipid oxidation, nucleotide-related compounds and aroma volatiles of Hanwoo brisket. Asian-Australasian Journal of Animal Sciences, 2018; 31(2): 293-300.

[14] $\mathrm{He} \mathrm{H} \mathrm{J,} \mathrm{Wu} \mathrm{D,} \mathrm{Sun} \mathrm{D} \mathrm{W.} \mathrm{Nondestructive} \mathrm{spectroscopic} \mathrm{and} \mathrm{imaging}$ techniques for quality evaluation and assessment of fish and fish products. Critical Reviews in Food Science and Nutrition, 2015; 55(6): 864-886.

[15] Ozdogan G, Lin X, Sun D W. Rapid and noninvasive sensory analyses of food products by hyperspectral imaging: Recent application developments. Trends in Food Science \& Technology, 2021; 111: 151-165.

[16] Balage J M, da Luz e Silva S, Gornide C A, Bonin M N, Figueira A C. Predicting pork quality using Vis/NIR spectroscopy. Meat Science, 2015; 108: 37-43.

[17] Moosavi-Nasab M, Khoshnoudi-Nia S, Azimifar Z, Kamyab S. Evaluation of the total volatile basic nitrogen (TVB-N) content in fish fillets using hyperspectral imaging coupled with deep learning neural network and meta-analysis. Scientific Reports, 2021; 11(1): $5094 . \quad$ doi: 10.1038/s41598-021-84659-y
[18] Shen T, Zhang C, Liu F, Wang W, Lu Y, Chen R, et al. High-throughput screening of free proline content in rice leaf under cadmium stress using hyperspectral imaging with chemometrics. Sensors, 2020; 20(11): 3229. doi: 10.3390/s20113229.

[19] Dixit Y, Al-Sarayreh M, Craigie C R, Reis M M. A global calibration model for prediction of intramuscular fat and $\mathrm{pH}$ in red meat using hyperspectral imaging. Meat Science, 2021; 181: 108405. doi: 10.1016/j.meatsci.2020.108405.

[20] Cheng W, Sun D W, Cheng J H. Pork biogenic amine index (BAI) determination based on chemometric analysis of hyperspectral imaging data. LWT- Food Science and Technology, 2016; 73: 13-19.

[21] Baek I, Lee H, Cho B K, Mo C, Chan D E, Kim M S. Shortwave infrared hyperspectral imaging system coupled with multivariable method for TVB-N measurement in pork. Food Control, 2020; 124: 107854. doi: 10.1016/j.foodcont.2020.107854.

[22] Elmasry G, Sun D W, Allen P. Non-destructive determination of water-holding capacity in fresh beef by using NIR hyperspectral imaging. Food Research International, 2011; 44(9): 2624-2633.

[23] Jiang H, Yoon S C, Zhuang H, Wang W, Lawrence K C, Yang Y. Tenderness classification of fresh broiler breast fillets using visible and near-infrared hyperspectral imaging. Meat Science, 2018; 139: 82-90.

[24] Dixit Y, Hitchman S, Hicks T M, Lim P, Wong C K, Holibar L, et al. Non-invasive spectroscopic and imaging systems for prediction of beef quality in a meat processing pilot plant. Meat Science, 2020; 181(1) 108410. doi: 10.1016/j.meatsci.2020.108410.

[25] Jiang H, Seung-Chul Y, Zhuang H, Wang W, Li Y, Lu C, et al. Non-destructive assessment of final color and $\mathrm{pH}$ attributes of broiler breast fillets using visible and near-infrared hyperspectral imaging: A preliminary study. Infrared Physics \& Technology, 2018; 92: 309-317.

[26] Achata E, Oliveira M, Esquerre C, Tiwari B K, O'Donnell C P. Visible and NIR hyperspectral imaging and chemometrics for prediction of microbial quality of beef Longissimus dorsi muscle under simulated normal and abuse storage conditions. LWT, 2020; 128: 109463. doi: 10.1016/j.lwt.2020.109463.

[27] Xiong Z, Sun D W, Pu H, Xie A, Han Z, Luo M. Non-destructive prediction of thiobarbituric acid reactive substances (TBARS) value for freshness evaluation of chicken meat using hyperspectral imaging. Food Chemistry, 2015; 179: 175-181.

[28] Aheto J H, Huang X, Tian X, Ren Y, Bonah E, Alenyorege E A, et al Combination of spectra and image information of hyperspectral imaging data for fast prediction of lipid oxidation attributes in pork meat. Journal of Food Process Engineering, 2019; 42(6): e13225. doi: $10.1111 /$ jfpe. 13225 .

[29] Kobayashi K, Matsui Y, Maebuchi Y, Toyota T, Nakauchi S. Near infrared spectroscopy and hyperspectral imaging for prediction and visualisation of fat and fatty acid content in intact raw beef cuts. Journal of Near Infrared Spectroscopy, 2010; 18(5): 301-315.

[30] Wang C, Wang S, He X, Wu L, Guo J. Combination of spectra and texture data of hyperspectral imaging for prediction and visualization of palmitic acid and oleic acid contents in lamb meat. Meat Science, 2020; 169: 108194. doi: 10.1016/j.meatsci.2020.108194.

[31] Jiang S, He H, Ma H, Chen F, Xu B, Liu H, et al. Quick assessment of chicken spoilage based on hyperspectral NIR spectra combined with partial least squares regression. Int J Agric \& Biol Eng, 2021; 14(1): 243-250.

[32] Barbin D F, Elmasry G, Sun D W, Allen P. Non-destructive determination of chemical composition in intact and minced pork using near-infrared hyperspectral imaging. Food Chemistry, 2013; 138(2-3): 1162-1171.

[33] Zhao P, Bai X W, Li Y K, Li Y, Lv C Y. A new method of data smoothing for scan-line point cloud in reverse engineering. Advanced Materials Research, 2014; 1006-1007: 352-355.

[34] Sharif M, Mohsin S, Jamal M J, Raza M. Illumination normalization preprocessing for face recognition. 2010 The $2^{\text {nd }}$ Coference on Environmental Science and Information Application Technology (ESIAT), Wuhan, China: IEEE, 2010; pp.44-47. doi: 10.1109/ESIAT. 2020.5567274 .

[35] Chu Y W, Tang S S, Ma S X, Ma Y Y, Hao Z, Guo Y M, et al. Accuracy and stability improvement for meat species identification using multiplicative scatter correction and laser-induced breakdown spectroscopy. Optics Express, 2018; 26(8): 10119-10127.

[36] Mishra P, Polder G, Gowen A, Rutledge D N, Roger, J M. Utilising variable sorting for normalisation to correct illumination effects in close-range spectral images of potato plants. Biosystems Engineering, 
2020; 197: 318-323.

[37] Rinnan A, Berg F V, Engelsen S B. Review of the most common pre-processing techniques for near-infrared spectra. Trends in Analytical Chemistry, 2009; 28(10): 1201-1222.

[38] Dai Q, Sun D W, Xiong Z, Cheng J H, Zeng X A. Recent advances in data mining techniques and their applications in hyperspectral image processing for the food industry. Comprehensive Reviews in Food Science \& Food Safety, 2014; 13(5): 891-905

[39] Kamruzzaman M, Makino Y, Oshita S. Rapid and non-destructive detection of chicken adulteration in minced beef using visible near-infrared hyperspectral imaging and machine learning. Journal of Food Engineering, 2016; 170: 8-15.

[40] Phetkaeo T, Klaithin R, Theanjumpol P, Kunasakdakul K, Thanapornpoonpong $\mathrm{S} N$, Vearasilp $\mathrm{S}$. Comparison of sample preparation methods on the infected corn seed detection by NIR spectroscopy. Chiang Mai University Journal of Natural Sciences, 2012; 11(1): 243-249.

[41] Huang X, Luo Y P, Xia L. An efficient wavelength selection method based on the maximal information coefficient for multivariate spectral calibration. Chemometrics and Intelligent Laboratory Systems, 2019; 194 103872. doi: 10.1016/j.chemolab.2019.103872.

[42] Chen Y, Shi R, Shu S, Gao W. Ensemble and enhanced PM10 concentration forecast model based on stepwise regression and wavelet analysis. Atmospheric Environment, 2013; 74: 346-359.

[43] Tang G, Huang Y, Tian K, Song X, Min S. A new spectral variable selection pattern using competitive adaptive reweighted sampling combined with successive projections algorithm. Analyst, 2014; 139(19): 4894-4902.

[44] Chen W, Zou J, Wan F, Fan Z, Yang D. Application of surface enhanced Raman scattering and competitive adaptive reweighted sampling on detecting furfural dissolved in transformer oil. AIP Advances, 2018; 8(3): 035204. doi: 10.1063/1.5012685.

[45] Andries J P M, Heyden Y V, Buydens L M C. Improved variable reduction in partial least squares modelling by global-minimum error uninformative-variable elimination. Analytica Chimica Acta, 2017; 982: 37-47.

[46] He H J, Wu D, Sun D W. Rapid and non-destructive determination of drip loss and $\mathrm{pH}$ distribution in farmed Atlantic salmon (Salmo salar) fillets using visible and near-infrared (Vis-NIR) hyperspectral imaging. Food Chemistry, 2014; 156: 394-401.

[47] Wang H, He H, Ma H, Chen F, Zhu R. LW-NIR hyperspectral imaging for rapid prediction of TVC in chicken flesh. Int J Agric \& Biol Eng, 2019; 12(3): 180-186.

[48] Zhu Y, He H, Jiang S, Ma H, Chen F, Xu B, et al. Mining hyperspectral data for non-destructive and rapid prediction of nitrite content in ham sausages. Int J Agric \& Biol Eng, 2021; 14(2): 182-187. 